\title{
Evaluations of polyelectrolyte multilayer membranes assembled by a dynamic layer-by-layer technique
}

\author{
Shulan $\mathrm{Ji}^{\mathrm{a}}$, Guojun Zhang ${ }^{\mathrm{a} *}$, Zhongzhou Liu ${ }^{\mathrm{b}}$, \\ Yuelian Peng ${ }^{\mathrm{a}}$, Zhan Wang ${ }^{\mathrm{a}}$ \\ ${ }^{a}$ College of Environmental and Energy Engineering, Beijing University of Technology, \\ Beijing 100022, PR China \\ Tel.+86(10)67392393; Fax+86(10)67391983; email: zhanggj@bjut.edu.cn \\ ${ }^{b}$ Research Center for Eco-Environmental Sciences, Chinese Academy of Sciences, \\ Beijing 100085, PR China
}

Received 6 August 2007; accepted revised 21 September 2007

\begin{abstract}
Self-assembly of polyelectrolyte multilayer membranes (PEMMs) by using dynamic layer-by-layer (LBL) adsorption technique was conducted in this study. The polyethyleneimine (PEI) and polyacrylic acid (PAA) were alternatively deposited on the hydrolyzed polyacrylonitrile (PAN) ultrafiltration membranes under a pressure of $0.1 \mathrm{MPa}$. The changes of membrane surfaces were observed by using atomic force microscopy. It was found that the regularity of the PEMMs was enhanced after the dynamic LBL assembly. The pervaporation separation behavior of various alcohol/water mixtures with the alcohols being $t$-butanol, 2-propanol and ethanol are compared with a relatively wide range of feed temperature and concentration.
\end{abstract}

Keywords: Polyelectrolyte multilayer membranes; Pervaporation separation; Alcohol/water mixtures; Atomic force microscopy

\section{Introduction}

Recent studies have shown that the alternating electrostatic layer-by-layer (LBL) adsorption of oppositely charged polyelectrolytes can be successfully applied to prepare ultrathin films

*Corresponding author. of very low, precisely controlled thickness. The membranes are suitable for the pervaportion separation of various alcohol/water mixtures. The previous studies of Tieke et al. [1-3] showed that a significantly large number of depositions of anionic/cationic polyelectrolytes are needed in order to achieve a high selectivity. It was

Presented at the Fourth Conference of Aseanian Membrane Society (AMS 4), 16-18 August 2007, Taipei, Taiwan. 
noted that as many as 60 bilayers had to be deposited to obtain membranes with sufficient properties in LBL adsorption process. In view of the fact that LBL is a time-consuming process, great efforts have also been made in developing new methods to reduce the number of deposition cycles required. For example, Sullivan and Bruening [4] combined the versatility of LBL adsorption with covalent interlayer cross-linking to assemble ultrathin polyimide pervaporation membranes. The most promising membranes obtained with 12.5 bilayers give a remarkable water-isopropanol selectivity of 6100 along with a flux of $2 \mathrm{~kg} \mathrm{~m}^{-2} \mathrm{~h}^{-1}$ for pervaporation of $90 \%$ isopropanol at $500^{\circ} \mathrm{C}$. Zhu et al. [5] proposed to reduce the deposition cycles by using a relatively dilute concentration of the polyelectrolytes in the first few cycles followed by depositions with more concentrated polyelectrolyte solutions. They also proposed to use a single-sided coating method to improve the membrane permeability. It was demonstrated by Zhu's study that a good permselectivity could be achieved with less than 10 cycles of deposition. We have reported [6] on the dynamic LBL adsorption technique, which would allow reduction of the deposition cycles. When the PAA/PEI pairs were deposited on a polyethersulfone (PES) ultrafiltration, the results suggested that a relatively high separation factor could be obtained with only 4 composite bilayers by using dynamic LBL process. More recently [7], the PAA/PEI pairs were successfully assembled on and the hydrolyzed polyacrylonitrile (PAN) ultrafiltration membranes support membrane under a certain pressure.

In this paper, the micrographs of the polyelectrolyte multilayer membranes assembled by a dynamic LBL process were observed by using an atomic force microscopy. The pervaporation separation behavior of various alcohol/ water mixtures with the alcohols being $t$-butanol, 2-propanol and ethanol are investigated with a relatively wide range of feed temperature and concentration.

\section{Experimental}

\subsection{Materials}

All the chemicals used in this study were of analytical grade. The PAA powder having average molecular weight of 4000,000 was obtained from Aldrich. Linear poly(ethyleneimine) (PEI) with the molecular weight of 60,000 was purchased from ACROS. The molecular weight cut-off of the support PAN UF membrane was 20,000 .

\subsection{Hydrolysis of PAN UF membrane}

The PAN UF membrane with an effective membrane area of $32 \mathrm{~cm}^{2}$ was hydrolyzed by immersing into the aqueous solutions of $2 \mathrm{~N}$ sodium hydroxide at $65^{\circ} \mathrm{C}$. After hydrolyzing for a $1 \mathrm{~h}$ period, the membranes was taken out and rinsed with de-ionized water until the $\mathrm{pH}$ values of the rinsed water reached about 7.0.

\subsubsection{Polyelectrolyte multilayer membrane preparation by dynamic $L B L$ process}

As previously reported [6-7], the preparation of polyelectrolyte multilayer membranes was carried out by using a $50 \mathrm{ml}$ stirred dead-ended filtration cell with approximately $79 \mathrm{~cm}^{2}$ membrane area. The hydrolyzed PAN UF membranes were loaded in a dead-ended filtration cell. The dynamic membrane was accomplished by filtrating the polyanion and polycation alternatively during a pre-determined period of filtration. The following steps were orderly carried out. (a) PEI solution was poured into the stirred cell and pressured under a pressure of $0.1 \mathrm{MPa}$ for a pre-determined period. (b) The membrane was taken out, briefly rinsed with de-ionized water for approximately $5 \mathrm{~min}$. and dried in an oven at $50^{\circ} \mathrm{C}$ for a pre-determined period. (c) PAA solution was poured into the stirred cell in which the dried membrane in (b) had been loaded, and was pressured through the membrane under a 
pressure of $0.1 \mathrm{MPa}$ by using a nitrogen cylinder. (d) The same way as (b). Steps (a) to (d) were repeated up to the pre-determined times. Upon the formation of polyelectrolyte multilayers membranes, they were stored for pervaporation experiments.

\subsubsection{Pervaporation experiments}

The membranes were characterized by the pervaporation of various alcohol/water mixtures $(95: 5 \mathrm{~g} / \mathrm{g})$ with the alcohols being $t$-butanol, 2-propanol and ethanol. The experimental apparatus and procedures for pervaporation have been described previously [6]. The membrane cell had an effective membrane area of $28.3 \mathrm{~cm}^{2}$. Fluxes were determined by measuring the weight of liquid collected in the cold traps during a certain time under steady-state conditions. The composition of the collected permeate was determined by gas chromatography (GC-14C,SHIMADZU).

\subsubsection{Characterization techniques}

The Atomic force microscopy (AFM) was conducted by using NanoScope IIIa System. Measurements were carried out in the air at room temperature.

\section{Results and discussion}

\subsection{Comparison of dynamic LBL and static $L B L$}

Table 1 summarizes the characteristic properties of polyelectrolyte deposition systems. A significantly large number of deposition cycles (e.g. 60) were often needed in order to achieve a relatively good selectivity during the conventional static LBL adsorption process. Although some researchers have made efforts to reduce the number of deposition cycles required, over 10 cycles were commonly required. As a comparison, only 2.5 bilayers were assembled to achieve reasonable pervaporation membrane during the dynamic LBL adsorption process. In the case of pervaporation of 95 wt.\% 2-propanol/ water mixture, the PEMMs had a separation factor of 833 and a permeate flux of $338 \mathrm{~g} / \mathrm{m}^{2} \mathrm{~h}$ $\left(70^{\circ} \mathrm{C}\right)$. Obviously, the deposition cycles were significantly reduced since the non-porous separation bilayers could be formed within a short period under the pressure. This suggested the adsorption between polycation and polyanion could be enhanced by extern force although the electrostatic force was the dominate formation reason. The substantial reduction of the number of polyion depositions would possibly make the manufacturing of the self-assembly polyelectrolyte composite membranes practically viable.

\subsection{Separation of different alcohol/water mixtures}

In Fig. 1a and b, the separation performances of alcohol/water mixtures with the alcohols being $t$-butanol, and 2-propanol or ethanol are compared. Fig. 1 suggested that the separation increased with the increase in molecular size of alcohol. Obviously, the higher separation factor could be obtained when the strength of the hydrogen bonding decreases with increasing number of carbon atoms in the alcohol. Meanwhile, hydrophobic alcohol molecules are more strongly rejected by the hydrophilic pores of the membrane. Fig. 2 also shows the pervaporation membranes with good performances were achieved by using the dynamic LBL technique even the selectivity declined rapidly with increasing water content in feed solution. Moreover, these membranes are really suitable for the separation of different alcohol/water mixtures within a relatively wide concentration range. Even in the case of separation of ethanol/water system, the permeate always contained a water content of more than 95 wt.\%. 


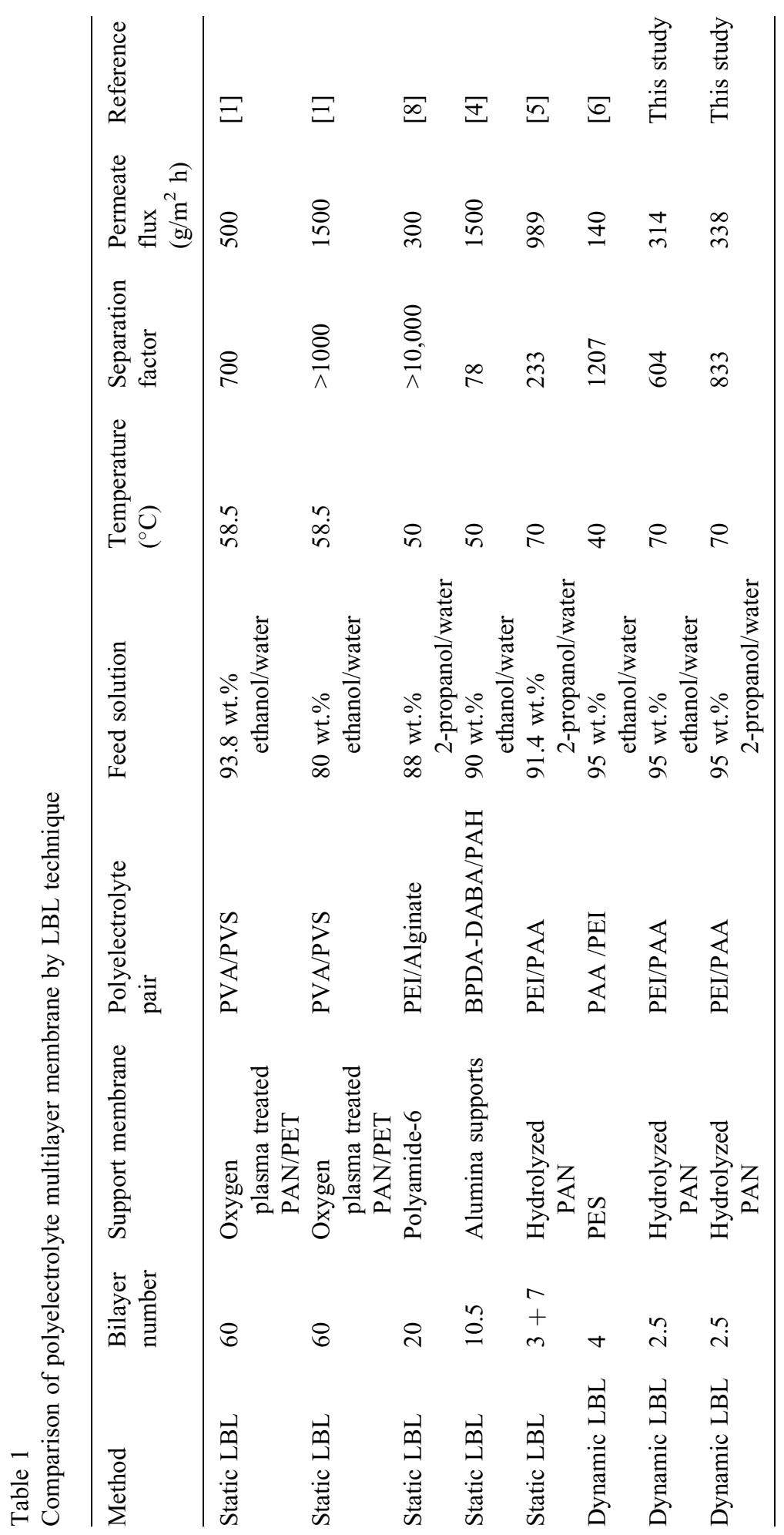




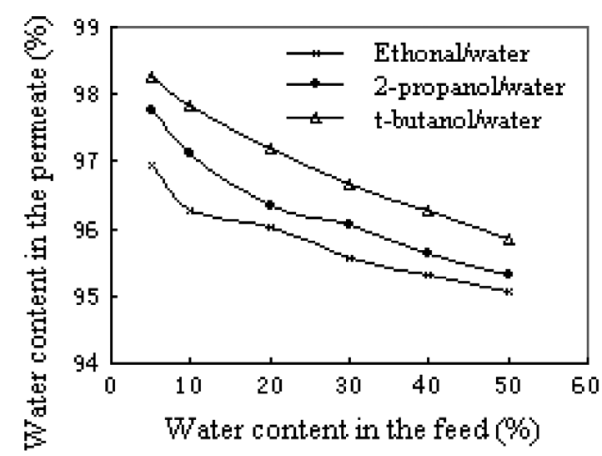

(a)

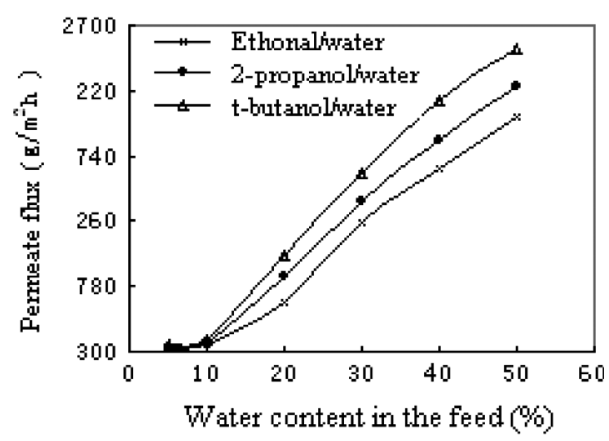

(b)

Fig. 1. (a) Dependence of water content in permeate (a) and total permeate flux (b) on water content in alcohol/water feed mixture for a PEI/PAA membrane assembled by dynamic LBL technique (Hydrolysis conditions for PAN support membrane: hydrolysis temperature: $65^{\circ} \mathrm{C}$, Hydrolysis time: $60 \mathrm{~min}$, Preparative conditions: 2.5 bilayerss, dynamic pressure: $0.1 \mathrm{Mpa}$, 20 min filtration time, 0.05 wt.\% PAA aqueous solution, 0.25 wt. $\%$ PEI-ethanol solution, $25^{\circ} \mathrm{C}$. Pervaporation conditions: feed temperature $70^{\circ} \mathrm{C}$, down-stream pressure $100 \mathrm{~Pa}$ ).

\subsection{AFM observations}

The AFM images were compared before and after assembling PEI/PAA pairs. Fig. 2 shows that polyion chains are adsorbed anisotropically on the surface. The membrane surface became much smoother after assembling the polyion pairs. Accordingly, the roughness decreased from $4.597 \mathrm{~nm}$ to $2.925 \mathrm{~nm}$. This suggested that the regularity of the PEMMs was enhanced after the

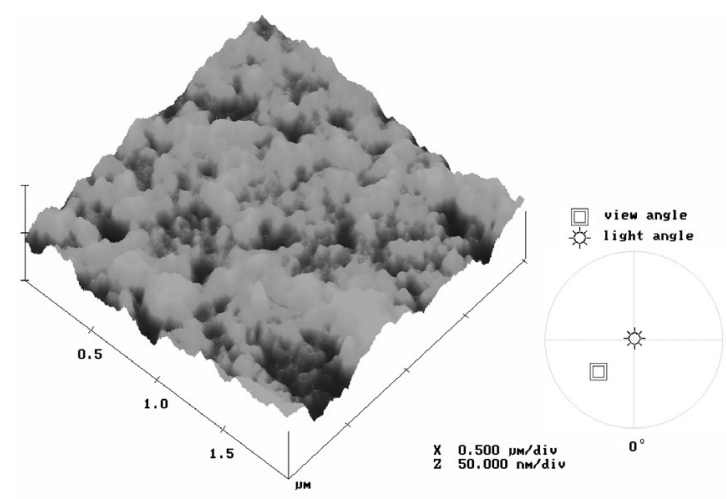

(a)

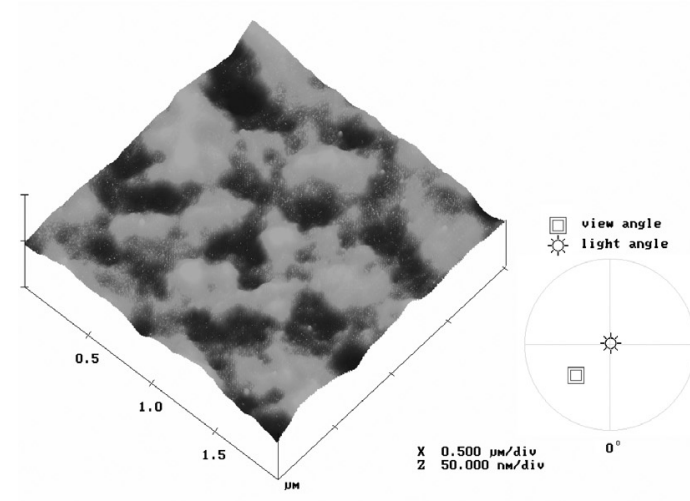

(b)

Fig. 2. AFM images (a) before assembling and (b) after assembling.

dynamic LBL assembly. In addition to the uniformity and denseness, the regularity of layerby-layer structural order might play a significant role in improving the pervaporation capability of membranes obtained. The AFM results further confirmed that the improvements of membrane surface regularity may be beneficial to high pervaporation performance after the dynamic LBL assembly.

\subsection{Stability of the PEEMs}

These experiments were intended to examine the operation stability of the PEEMs assembled by dynamic LBL adsorption process. The 


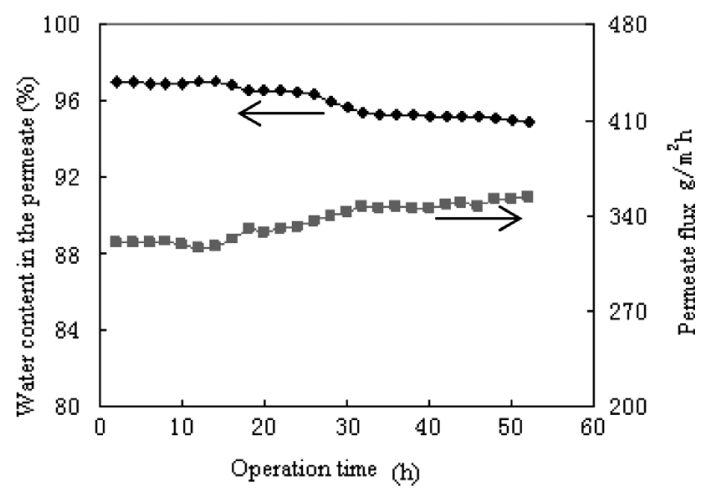

Fig. 3. Test of operation stability of complex membrane (Preparative conditions: dynamic pressure: $0.1 \mathrm{MPa}$, $[\mathrm{PAA}] /[\mathrm{PEI}]=1 / 5,25^{\circ} \mathrm{C}$; pervaporation conditions: feed temperature $70^{\circ} \mathrm{C}$, EtOH content in feed solution 95 wt.\%, down-stream pressure $100 \mathrm{~Pa}$ ).

pervaporation separation of $95 \%$ ethanol/water system was intermittently conducted for $52 \mathrm{~h}$. The results are summarized in Fig. 3.

These experiments demonstrate clearly the relatively good long-term stability of the polyelectrolyte multilayer membranes. Although the separation performance changed a little over the operation time, the water content in the permeate still kept over $95 \%$.

\section{Conclusions}

In this paper, the performance of the PEMMs assembled by a dynamic LBL adsorption technique was evaluated and compared with the PEEMs assembled by conventional static LBL. The pervaporation separation of various alcohol/water mixtures suggested that the PEEMs with only 2.5 bilayers had a good capacity with a relatively wide range of feed temperature and concentration. The AFM show that the regularity of the membrane surface was improved after the dynamic LBL assembly. In addition, the PEEMs with PAA/PEI pairs deposited on hydrolyzed PAN membranes were relatively stable through $52 \mathrm{~h}$ pervaporation separation of $95 \%$ ethanol/ water mixture.

\section{Acknowledgement}

This work was supported by the Beijing Natural Science Foundation (No. 8071001), the Beijing NOVA Programme (No. 2006B13), the Special Funding Project of the Beijing Municipal Commission of Education, PR, China (No. 05005999200601), the Excellent Project for the Returned Overseas Chinese Scholars, Beijing Municipal Bureau of Personnel (No. 38005013200701) and the National Basic Research Program of China (No.2003CB615701).

\section{References}

[1] Ali Toutianoush, Lutz Krasemann, Bernd Tieke, Polyelectrolyte multilayer membranes for pervaporation separation of alcohol/water mixtures, Colloids Surf., A: Physicochem Engg Aspects, 198-200 (2002) 881-889.

[2] L. Krasemann, A. Toutianoush and B. Tieke, Selfassembled polyelectrolyte multilayer membranes with highly improved pervaporation separation of ethanol/water mixtures, J. Membr. Sci., 181 (2001) 221-228.

[3] A. Toutianoush and B. Tieke, Pervaporation separation of alcohol/water mixtures using self-assembled polyelectrolyte multilayer membranes of high charge density, Mater. Sci. Eng., C 22 (2002) 459-463.

[4] D.M. Sullivan and M.L. Bruening, Ultrathin, crosslinked polyimide pervaporation membranes prepared from polyelectrolyte multilayers, J. Membr. Sci., 248 (2005) 161-170.

[5] Zhaoqi Zhu, Xianshe Feng and Alexander Penlidis, Self-assembled nano-structured polyelectrolyte composite membranes for pervaporation, Mater. Sci. Eng., C 26 (2006) 1-8.

[6] Guojun Zhang, Weiliang Gu, Shulan Ji, Zhongzhou Liu, Yuelian Peng and Zhan Wang, Preparation of Polyelectrolyte multilayer membranes by dynamic layer-by-layer process for pervaporation separation of alcohol/water mixtures, J. Membr. Sci., 280 (2006) 727-733.

[7] Guojun Zhang, Haihong Yan, Shulan Ji and Zhongzhou Liu, Self-Assembly of Polyelectrolyte Multilayer Pervaporation Membranes by a Dynamic Layer-by-Layer Technique on a Hydrolyzed 
Polyacrylonitrile Ultrafiltration Membrane, J. Membr. Sci., 292 (2007) 1-8.

[8] Jochen Meier-Haack, Wolfgang Lenk, Dieter Lehmann and K. Lunkwitz, Pervaporation separation of water/alcohol mixtures using composite membranes based on polyelectrolyte multilayer assemblies[J]. J. Membr. Sci., 184 (2001) 233-243. 